Reprod. Nutr. Dévelop., 1982, 22 (4), 583-596.

\title{
Biosynthèse de la carnitine chez les mammifères
}

\author{
A. $\mathrm{BACH}$
}

Laboratoire de Pathologie générale, Service de Médecine interne A, Hospices Civils, 67091 Strasbourg Cedex.

Summary. Carnitine biosynthesis in mammals.

The oxidation of long-chain fatty acids is carnitine-dependent; these fatty acids penetrate the mitochondrion to be oxidized only when they are bound to carnitine in the form of acyl-carnitines. To meet the need for carnitine, animals depend on both exogenous supplies and endogenous synthesis. The aim of the present paper is to review our knowledge of endogenous carnitine synthesis.

The precursors of carnitine are lysine and methionine but its true point of origin is trimethyllysine. This molecule is either obtained from the diet or is synthesized in the body from L-lysine (bound to protein) which is methylated 3 consecutive times by an Sadenosyl-methionine. Trimethyllysine is transformed into hydroxy-trimethyllysine, then into trimethylaminobutyraldehyde and finally into trimethylaminobutrate (or $\gamma$-butyrobetaine). The $\gamma$-butyrobetaine is hydroxylated into carnitine. This reaction chain only functions well when three vitamins - ascorbic acid, pyridoxin and niacin - are present.

Studies on rat have shown that skeletal muscle, heart, intestines, testis, and especially kidneys, insure the transformation of trimethyllysine into $\gamma$-butyrobetaine but that only the testis, and especially liver, can hydroxylate $\gamma$-butyrobetaine into carnitine. However, in rat the relative importance of the kidneys and liver in total carnitine synthesis has not yet been determined. The situation is the same in man, although it has been proven that human brain and kidneys, as liver, have $\gamma$-butyrobetaine hydroxylase.

It is known that the rate of carnitine synthesis depends on three factors - the amount of trimethyllysine available, the rate of $\gamma$-butyrobetaine transfer to tissue(s) hydroxylating it and $\gamma$-butyrobetaine hydroxylase activity. Moreover, it appears that carnitine synthesis is not slowed down by prolonged fasting, that it does not completely cover body needs during the first postnatal days, and that it does not decrease in two patients with systematic carnitine deficiency.

\section{Introduction.}

La carnitine ( $\beta$-hydroxy- $\gamma$-triméthylaminobutyrate) est un constituant que l'on rencontre dans les tissus de la plupart des eucaryotes, qu'il s'agisse de microorganismes ou de plantes, de vertébrés ou d'invertébrés (Fraenkel, 1953 ; Fraenkel et Friedman, 1957). Découverte dans le muscle de bœuf en 1905, sa structure a été établie en 1927 (voir Fraenkel et Friedman, 1957). C'est vers les années 1950 que Fraenkel (1953) montra que la carnitine est un facteur de croissance indispensable pour le ver de farine, Tenebrio molitor (*). Peu de

(*) Fraenkel avait dénommé ce facteur vitamine $B_{T}$. Par la suite, celle-ci se révéla être de la carnitine. 
temps après, on commença à entrevoir la fonction essentielle de la carnitine dans le transport des acides gras à travers la membrane mitochondriale (Fritz, 1955). On sait maintenant que les acides gras et acyls-CoA à longues chaînes sont incapables de pénétrer dans la mitochondrie, pour y être oxydés, sans l'aide de la carnitine, d'enzymes (les acyl-carnitine transferases) et d'un système de transport complexe (l'acyl-carnitine translocase). Enfin, à partir de 1973, on prit conscience de l'existence d'une déficience en carnitine musculaire dans certaines myopathies (Engel et Angelini, 1973 ; Karpati et al., 1975).

Si chez la plupart des mammifères une partie de la carnitine est apportée par l'alimentation, la plus grande partie est d'origine endogène. Une bonne connaissance des différentes étapes de sa biosynthèse, ainsi que de la localisation tissulaire et de la régulation de cette synthèse, est indispensable pour entreprendre des investigations dans le métabolisme aberrant de la carnitine, tel qu'on peut l'observer dans les myopathies. Rapporter ce que l'on sait actuellement dans ces domaines est le but de cette revue.

\section{Les étapes de la synthèse de la carnitine.}

\section{La $\gamma$-butyrobétaine.}

II y a plus de 50 ans, Linneweh (1929) signalait que I'administration de $\gamma$ butyrobétaine (4-triméthylaminobutyrate) entraîne chez le chien une augmentation de l'excrétion urinaire de carnitine. Les travaux de Lindstedt et Lindstedt (1961) et de Bremer (1962), avec de la $\gamma$-butyrobétaine marquée, ont montré que celle-ci était rapidement transformée en carnitine par le rat et qu'une fraction importante de la radioactivité était retenue par le muscle, le foie et le rein, sous forme de carnitine.

Ultérieurement, on a constaté que la $\gamma$-butyrobétaine est hydroxylée sur le $3^{3}$ carbone. La réaction est catalysée par une $\gamma$-butyrobétaine hydroxylase (Lindstedt et Lindstedt, 1961, 1962, 1970 ; Lindstedt, 1967 ; Englard, Horwitz et Mills, 1978), nécessitant la présence d'oxygène moléculaire, $d^{\prime} \alpha$-cétoglutarate et de $\mathrm{Fe}^{2+}$. L'enzyme est activée par l'acide ascorbique.

\section{Lysine et méthionine, précurseurs de la carnitine.}

Un deuxième pas dans l'élucidation du mécanisme de la biosynthèse de la carnitine a été franchi, lorsqu'on a découvert que deux acides aminés essentiels, la lysine et la méthionine, en étaient des précurseurs.

a) Quelques observations. - Une déficience en lysine et en carnitine induite par une alimentation n'apportant comme protéine que du gluten, conduit chez le rat à un ralentissement de la croissance et à une chute de la teneur en carnitine dans le sang et les muscles squelettiques et cardiaques (Tanphaichitr, Horne et Broquist, 1971 ; Tanphaichitr et Broquist, 1973a ; Borum et Broquist, 1977b ; Khan et Bamji, 1979). L'oxydation du palmitate est ralentie dans le cœur (Khan et Bamji, 1979). Dans les tissus musculaires, on observe une accumulation lipidique anormale qui s'explique (Tanphaichitr, Zaklama et Broquist, 1976) 
partiellement du moins, par un manque de carnitine nécessaire au transport intramitochondrial - étape intermédiaire indispensable avant l'oxydation - des acides gras. L'addition de carnitine au régime alimentaire fait régresser la majorité des troubles.

Par ailleurs, Khairallah et Wolf (1965) ont montré que l'addition de carnitine à un régime pauvre en méthionine conduit à une reprise de la croissance et à une diminution de la teneur lipidique du foie.

Certaines formes sévères de malnutrition protéique (Mikhail et Mansour, 1976 ; Khan et Bamji, 1977) se traduisent chez l'homme par une chute de la teneur sanguine en carnitine. En effet, ces sujets consomment exclusivement certains végétaux, pauvres non seulement en carnitine, mais également en lysine et en méthionine (Mitchell, 1978).

b) Les précurseurs de la carnitine. - II a d'abord été établi que les trois groupes méthyles de la carnitine proviennent de la méthionine (Bremer, 1961 ; Wolf et Berger, 1961 ; Cox et Hoppel, 1973a ; Horne et Broquist, 1973, Tanphaichitr et Broquist, 1973b).

Un peu plus tard, d'abord chez Neurospora crassa (Horne, Tanphaichitr et Broquist, 1971 ; Horne et Broquist, 1973), puis chez le rat (Tanphaichitr, Zaklama et Broquist, 1973 ; Tanphaichitr et Broquist, 1973a, b ; Cox et Hoppel, 1973a), on a montré que la L-lysine était incorporée dans la carnitine : ce sont les carbones 3, 4, 5 et 6 de la lysine qui fournissent la chaîne carbonée de la carnitine, l'E- $\mathrm{N}$ de la lysine procurant l'azote de cette molécule (Tanphaichitr, Horne et Broquist, 1971 ; Cox et Hoppel, 1973b ; Horne et Broquist, 1973.)

\section{Du 6-N-triméthyl-L-lysine à la $\gamma$-butyrobétaine.}

La clé de la biosynthèse de la carnitine se trouve dans la découverte de l'origine de la $\gamma$-butyrobétaine. Et ce n'est qu'en 1973, que l'on a compris le rôle primordial joué par le 6-N-triméthyl-L-lysine ( $\mathrm{Me}_{3}$-lysine). Chez Neurospora (Horne et Broquist, 1973 ; Kaufman et Broquist, 1977 ; Broquist, 1980) comme chez le rat, I'utilisation de la $\mathrm{Me}_{3}$-lysine marquée au carbone 14 , a indiqué que cette substance est un précurseur de la $\gamma$-butyrobétaine et de la carnitine. Chez le rat in vivo, la $\mathrm{Me}_{3}$-lysine est convertie en 5 métabolites qui ont été isolés à partir des urines : la carnitine, la $\gamma$-butyrobétaine, la 3-hydroxy-6-N-triméthyl-L-lysine, ainsi que deux autres produits qui ne sont pas des intermédiaires dans la synthèse de la carnitine (Hoppel, Novak et Cox, 1976 ; Hoppel, Cox et Novak, 1980). La Me $\mathbf{3}^{-}$ lysine est donc bien un précursẹur de la carnitine. Cette expérience montre de plus, et cela a été confirmé par ailleurs (Hulse, Ellis et Henderson, 1978 ; Sachan et Hoppel, 1980), que la $\mathrm{Me}_{3}$-lysine est hydroxylée sur le $3^{\mathrm{e}}$ carbone en 3hydroxy-6-N-triméthyl-L-lysine en présence d'une 6-N-triméthyllysine-3hydroxylase. Cet enzyme nécessite la présence $d^{\prime} \mathrm{O}_{2}, d^{\prime} \alpha$-cétoglutarate, de $\mathrm{Fe}^{2+}$ et $d^{\prime}$ ascorbate.

Hochalter et Henderson (1976) ont signalé que l'administration de $\mathrm{Me}_{3}$-lysine entraînait de plus l'élimination urinaire de glycocolle (en réalité sous forme d'hippurate, car les auteurs fournissent en plus de la $\mathrm{Me}_{3}$-lysine, du benzoate de $\mathrm{Na}$ ). 
Cette observation s'explique par l'étape suivante : sous l'action d'une 3-hydroxy6-triméthyl-lysine aldolase, la 3-hydroxy-triméthyl-L-lysine est clivée entre les carbones 2 et 3 avec production de 4- $\mathrm{N}$-triméthylaminobutyraldéhyde d'une part et libération de glycocolle d'autre part (Kaufman et Broquist, 1977; Broquist, 1980 ; Rebouche, 1980 ; Henderson, Hulse et Henderson, 1980). Cette réaction peut également se faire en présence d'une sérine transhydroxyméthylase (Henderson, Hulse et Henderson, 1980), avec le phosphate de pyridoxine comme cofacteur.

Finalement, cet aldéhyde est oxydé en 4- $\mathrm{N}$-triméthyl-aminobutyrate ou $\gamma$ butyrobétaine sous l'influence soit d'une $\gamma$-triméthyl-aminobutyraldéhyde deshydrogénase (en présence de $\mathrm{NAD}^{+}$), soit d'une aldéhyde oxydase (en présence de $\mathrm{FAD}^{+}$) (Hulse et Henderson, 1980; Rebouche, 1980; Rebouche et Engel, 1980c).

\section{Synthèse de la triméthyl-L-lysine.}

C'est finalement l'étape qui conduit de la lysine et de la méthionine à la triméthyllysine qui est encore en l'état actuel des connaissances, la plus discutée. La triméthyllysine se rencontre dans tous les organismes vivants étudiés, soit sous forme libre (en très faibles quantités dans le sang et les urines de l'homme), soit sous forme liée à des protéines (histone du thymus; cytochrome $\mathrm{C}$ de la levure ou du germe de blé ; myosine du muscle squelettique; actine de l'amibe) (Paik et Kim, 1975).

Chez Neurospora crassa, Rebouche (1976, 1980) et Broquist (1980) ont constaté la méthylation par étape d'une lysine libre en triméthyllysine : le donneur de méthyle est une S-adénosyl-L-méthionine; une $\mathrm{S}$-adénosyl-Lméthionine : 6-N-L-lysine méthyltransferase (Borum et Broquist, 1977a) catalyse les 3 méthylations successives; la monométhyllysine et la diméthyllysine sont les intermédiaires de cette réaction.

Mais chez les mammifères, il n'a jamais été possible de mettre en évidence une méthylation de la lysine libre. Contrairement à ce qui se passe chez Neurospora, les organismes supérieurs sont incapables de fabriquer de la carnitine à partir de la mono- ou de la diméthyllysine (Labadie, Dunn et Aronson, 1976) : la méthylation de ces deux substances est impossible sous leur forme libre. D'où la proposition de Paik et Kim (1975), la triméthyllysine destinée à la biosynthèse de la carnitine dérive d'une lysine liée aux chaînes polypeptidiques. Non seulement la lysine doit être liée aux protéines pour être méthylée (Krzysik, Vergnes et McManus, 1971), mais de plus, elle doit être totalement méthylée en triméthyllysine, avant d'être libérée par dégradation protéolytique. Paik et Kim (1971, 1975, 1977) et Paik, Lee et Lawson (1971) ont isolé une protéine méthylase III (Sadenosyl-méthionine protéine-lysine méthyltransferase) qui méthyle successivement la lysine liée aux protéines en mono-, puis di- et enfin triméthyl-L-lysine.

$S^{\prime} i l$ est acquis chez les mammifères que la triméthylysine provenant de la dégradation lysosomale des protéines (Labadie, Dunn et Aronson, 1976; Anonyme, 1978) quitte le lysosome et est utilisée dans la synthèse de carnitine, il n'est pas prouvé que cette source de précurseur soit la seule existante. 
5. Schéma général.

Dans la figure 1, nous avons indiqué les différentes étapes de la biosynthèse de la carnitine telle qu'on se la représente actuellement. Celle-ci nécessite donc à la fois 2 acides aminés essentiels, lysine et méthionine et 3 vitamines : acide ascorbique (activateur des 2 hydroxylases), pyridoxine (cofacteur de la sérine transhydroxyméthylase) et acide nicotinique (pour la synthèse du NAD nécessaire) (Anonyme, 1978).

Une partie importante de la carnitine est acylée en acyl-carnitine. Suivant la longueur de la chaîne carbonée de l'acide gras concerné, on distingue les acylcarnitines à chaînes longues (perchloroinsolubles), les acyl-carnitines à chaînes courtes (perchlorosolubles) et l'acétyl-carnitine (perchlorosoluble). La limite entre les deux premiers groupes d'acyl-carnitines se situe suivant les auteurs au niveau du decanoate ou du dodecanoate. Jusqu'à présent, trois enzymes catalysant cette acylation ont été isolées: la palmityl-carnitine transferase, l'octanoylcarnitine transferase et l'acétyl-carnitine transferase (Solberg, 1971).

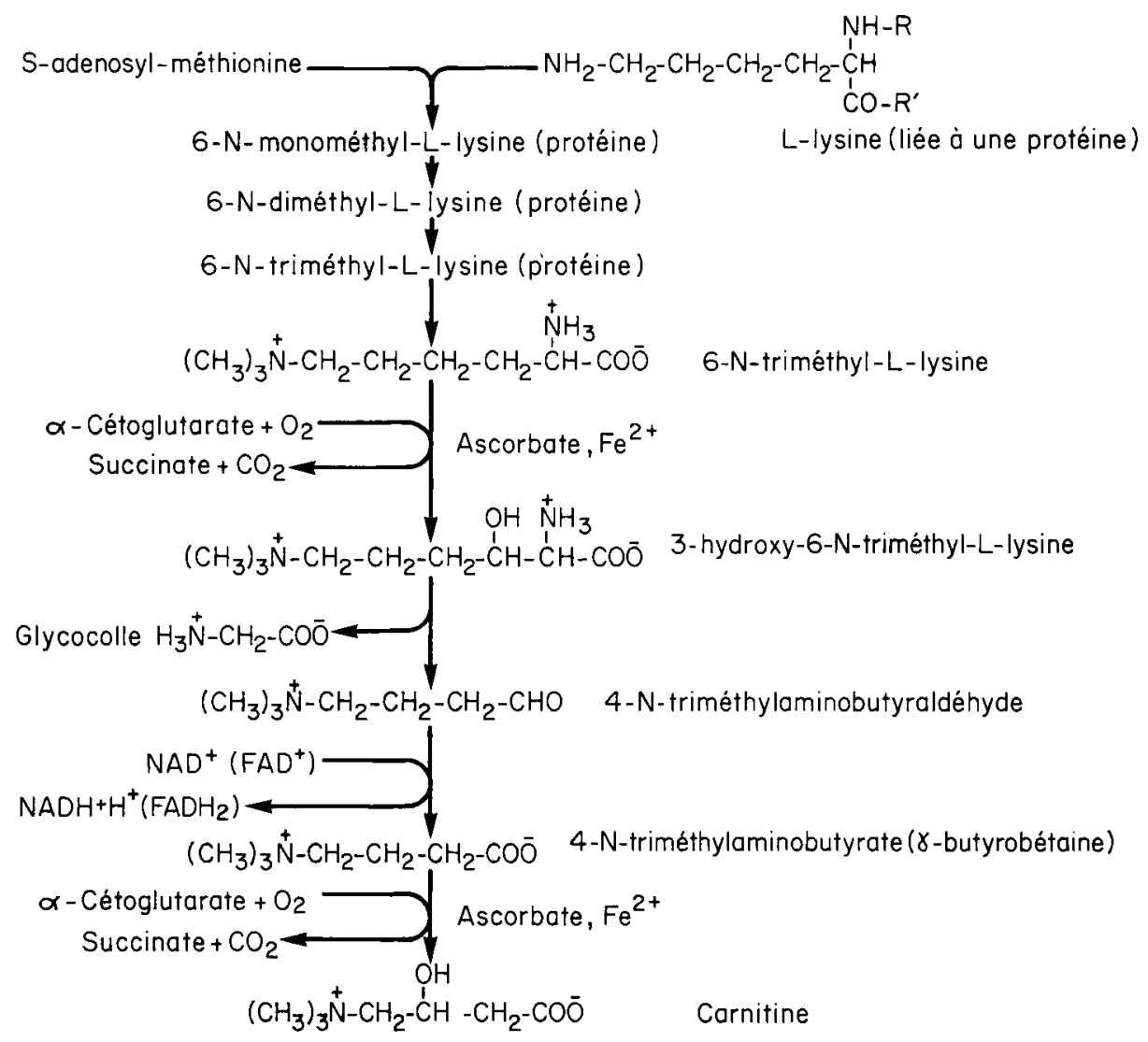

FIG. 1. - Biosynthèse de la carnitine. 


\section{Localisation tissulaire de la synthèse de la carnitine.}

\section{Synthèse chez le rat.}

Différents laboratoires ont montré chez le rat que le foie, le tissu adipeux, les muscles squelettiques et cardiaque, les reins, l'intestin, les testicules et l'épididyme étaient capables d'assurer toute la séquence de réactions conduisant de la triméthyllysine à la $\gamma$-butyrobétaine (Broquist et Tanphaichitr, 1973 ; Cox et Hoppel, 1974 b ; Haigler et Broquist, 1974 ; Tanphaichitr et Broquist, 1974 ; Casillas et Erickson, 1975: Zaspel et Henderson, 1979; Zaspel, Sheridan et Henderson, 1980) (fig. 2).

30 minutes après une injection intraveineuse de $\mathrm{Me}_{3}$-lysine marquée, une grande partie de la radioactivité est localisée dans les reins -20 fois plus que dans le foie (Carter et Frenkel, 1979 ; Frenkel et Carter, 1980) - sous forme de triméthyllysine, d'hydroxytriméthyllysine et de $\gamma$-butyrobétaine. Dans le foie, on trouve en plus de la carnitine marquée (Cox et Hoppel, 1974a, b ; Haigler et Broquist, 1974 ; Carter et Frenkel, 1979 b). Par ailleurs, le foie des rats binéphrectomisés contient 5 fois moins de radioactivité que celui des témoins (Carter et Frenkel, 1979 ; Frenkel et Carter, 1980). Ces observations suggèrent non seulement un prélèvement rénal préférentiel de la triméthyllysine (Carter et Frenkel, 1979 ; Zaspel, Sheridan et Henderson, 1980 ; Nelson et al., 1981), mais également la nécessité de la présence des reins pour permettre l'accumulation hépatique de $\gamma$ butyrobétaine et de carnitine. A propos du prélèvement rénal de la triméthyllysine, des travaux in vitro (Zaspel et Henderson, 1979 ; Zaspel, Sheridan et Henderson, 1980) ont révélé que celui-ci ne se faisait pas bien. On pense que c'est par réabsorption tubulaire que le rein récupère la triméthyllysine circulante $d^{\prime}$ origine exogène ou endogène.

Dans le rein, la triméthyllysine est rapidement convertie en $\gamma$-butyrobétaine. Frenkel et Carter (1980) affirment que les reins sont responsables pour une grande partie de la production de $\gamma$-butyrobétaine, même s'ils n'exciuent pas une possible contribution de la part des autres tissus.

Quant à l'hydroxylation de la $\gamma$-butyrobétaine, elle paraît limitée au foie et, dans une bien moindre mesure, aux testicules (Haigler et Broquist, 1974 ; Cox et Hoppel, 1974b ; Tanphaichitr et Broquist, 1974). Seuls ces deux tissus paraissent posséder la $\gamma$-butyrobétaine hydroxylase (Erfle, 1975 ; Englard et Carnicero, 1978 ; Cederblad et al., 1979).

Tous ces travaux sur le rat ont conduit à la théorie suivante : la $\gamma$ butyrobétaine, fabriquée principalement par le rein, quitte cet organe et est transportée au foie via la circulation sanguine (Tanphaichitr et Broquist, 1974 ; Bøhmer, 1974 ; Christiansen et Bremer, 1976 ; Carter et Frenkel, 1979). Contrairement à la triméthyllysine, la $\gamma$-butyrobétaine est rapidement captée par le foie (Zaspel et Henderson, 1979 ; Zaspel, Sheridan et Hendersen, 1980). Elle est transportée à travers la membrane plasmique contre un gradient de concentration (Christiansen et Bremer, 1976). Le foie est ainsi le site privilégié de la synthèse de carnitine. Le fait que l'on n'observe aucune conversion de la $\gamma$ butyrobétaine en carnitine chez le rat dont le foie est exclu de la circulation (Bøhmer, 1974) confirme cette théorie. 
Pour Zaspel, Sheridan et Henderson (1980), les choses se passent un peu différemment. Certes, le rein est le lieu privilégié de la synthèse de $\gamma$ butyrobétaine à partir de la triméthyllysine, mais exclusivement de celle apportée par l'alimentation. En effet, ils ont montré que le foie ne prélève pas la $\mathrm{Me}_{3^{-}}$ lysine du plasma ou du liquide de perfusion. Pour eux, la carnitine est surtout produite à partir de la triméthyllysine endogène (Labadie, Dunn et Aronson, 1976), libérée au cours du turnover des protéines hépatiques; le foie étant le lieu privilégié du turnover des protéines contenant de la $\mathrm{Me}_{3}$-lysine (Hulse et Henderson, 1980). Ainsi, le foie serait le lieu de la synthèse intégrale de la carnitine, les reins ne jouant qu'un rôle secondaire, proportionnel à la part de triméthyllysine qu'ils produisent. Des travaux supplémentaires sont donc encore nécessaires pour départager les rôles respectifs du rein et du foie dans la biosynthèse de la carnitine chez le rat.

La carnitine synthétisée par le foie quitte ce tissu. Pour Bøhmer (1974), cette libération est passive, car il a observé que le rapport acétylcarnitine/carnitine perchlorosoluble totale est le même dans le sang et le foie.

\section{Synthèse chez l'homme.}

Mais ces dernières années, on s'est rendu compte que la localisation tissulaire de la $\gamma$-butyrobétaine hydroxylase variait énormément d'une espèce à l'autre. Si l'enzyme est absent du rein de rat, mais aussi de cobaye, de souris et de chien, il a été retrouvé dans le foie et le rein de hamster, de lapin, de chat, de mouton et de singe (Erfle, 1975 ; Englard et Carnicero, 1978 ; Englard, Horwitz et Mills, 1978 ; Englard, 1979) (fig. 2). Chez le mouton, le muscle squelettique aurait une activité $\gamma$-butyrobétaine hydroxylasique, le muscle cardiaque non (Cederblad et al., 1979). Chez l'homme enfin, l'enzyme a été trouvée dans le foie, mais aussi dans le rein et dans le cerveau (Englard, 1979 ; Rebouche, 1980 ; Rebouche et Engel, 1980c ; Anonyme, 1981). Comme l'activité de l'enzyme rénal est environ 3,5 fois supérieure à celle du foie, on doit admettre que chez l'homme, le rein joue un rôle non négligeable dans la synthèse de la carnitine (Rebouche et Engel, 1980c).

Les résultats présentés en 1980 par Rebouche et Engel (1980a) indiquent que chez l'homme et chez le rat, le rein accumule la $\mathrm{Me}_{3}$-lysine circulante. Celle-ci est transformée en $\gamma$-butyrobétaine chez le rat et en carnitine chez l'homme. Dans les deux espèces, le produit formé est finalement libéré dans la lumière des tubules rénaux, d'où il est soit réabsorbé dans la circulation sanguine pour être distribué aux autres tissus, soit excrété. Mais pour les deux auteurs, il est théoriquement possible que la transformation de la $\mathrm{Me}_{3}$-lysine en $\gamma$-butyrobétaine se fasse dans le tissu où la $\mathrm{Me}_{3}$-lysine est produite (foie, mais aussi muscle, cœur, rein, cerveau). Ce n'est pas alors la $\mathrm{Me}_{3}$-lysine qui est relâchée dans la circulation générale, mais la $\gamma$-butyrobétaine, voire la carnitine. Cette $\gamma$-butyrobétaine est prélevée par le foie et le rein (Zaspel et Henderson, 1979). Dans cette hypothèse et compte-tenu d'une part de l'activité de la $\gamma$-butyrobétaine hydroxylase dans ces deux organes et $d^{\prime}$ 'autre part de leur masse respective, foie et reins auraient sensiblement une égale importance dans la biosynthèse de la carnitine chez I'homme (Rebouche et Engel, 1980a). 


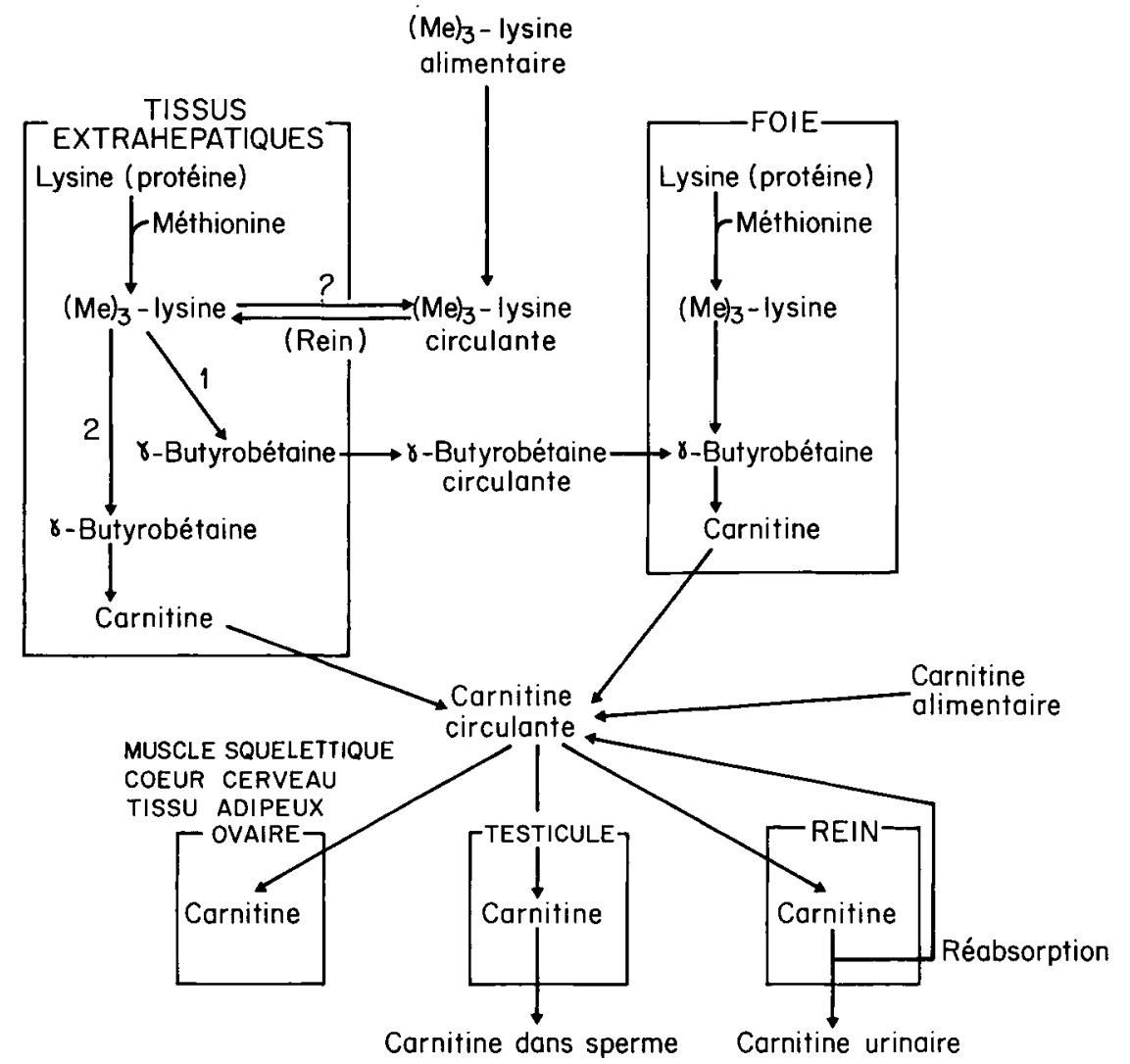

FIG. 2. - Localisation tissulaire du métabolisme de la carnitine. 1. Voie empruntée dans le coeur, le muscle squelettique, l'intestin, le tissu adipeux et surtout le rein de rat, de la souris, du cobaye et du chien. 2. Voie empruntée dans le testicule de rat et d'homme et dans le rein et le cerveau de l'homme, du chat, du hamster, du lapin et du singe Rhésus markey.

$(\mathrm{Me})_{3}$-lysine $=6-\mathrm{N}$-triméthyl-L-lysine

\section{Régulation de la synthèse de carnitine.}

1. Synthèse dans l'organisme sain.

Si la chaîne réactionnelle conduisant à la biosynthèse de la carnitine est maintenant bien élucidée, l'importance et les mécanismes de régulation de celleci sont relativement mal connus. C'est ainsi que l'on ne sait pas si la synthèse endogène est affectée par une variation de l'apport exogène de carnitine. La seule chose que l'on sache, c'est que les tissus d'animaux nourris avec une alimentation déficiente en choline et en méthionine, incorporent 15 fois plus la méthionine marquée dans la carnitine que ceux prélevés sur des rats témoins (Strength, Yu et Davis, 1965). Dans le même ordre d'idée, il a été prouvé que l'élimination urinaire de carnitine varie dans le même sens que la quantité de carnitine alimentaire (Mitchell, 1978). 
Selon Tanphaichitr et Broquist (1973b), 0.1 p. 100 seulement des besoins alimentaires quotidiens en lysine et en méthionine est utilisé pour la synthèse de carnitine. Mais si l'un de ces deux acides aminés se trouve à un taux limite dans le régime alimentaire, il est possible que la synthèse de carnitine en soit d'autant diminuée (Broquist et Borum, 1977). Le problème se pose probablement avec plus d'acuité pour la méthionine que pour la lysine. Si cette dernière manque totalement dans l'alimentation, l'organisme économise ce nutriment (Broquist et Borum, 1977 ; Anonyme, 1978), et l'on n'observe qu'une faible diminution de la carnitine dans le plasma et les muscles cardiaque et squelettiques (Borum et Broquist, 1977b).

Des études chez le rat suggèrent que l'activité de la $\gamma$-butyrobétaine hydroxylase est le facteur limitant dans la synthèse de la carnitine : son substrat est en effet le seul intermédiaire qui $s^{\prime}$ accumule après administration de triméthyllysine (Cox et Hoppel, 1973b ; Tanphaichitr et Broquist, 1973b). Chez l'homme, Rebouche et Engel (1980c) pensent que trois paramètres déterminent la vitesse de synthèse : la quantité de triméthyllysine disponible, la vitesse de transfert de la $\gamma$-butyrobétaine au foie et au rein, enfin l'activité de la $\gamma$ butyrobétaine hydroxylase.

Deux des enzymes intervenant dans la chaîne réactionnelle conduisant à la carnitine, la triméthyl-L-lysine hydroxylase et la triméthylaminobutyrate hydroxylase, ont un besoin, certes non absolu, mais spécifique, d'acide ascorbique (Lindstedt, Lindstedt et Nordin, 1980). En I'absence de vitamine C, la vitesse des réactions que ces enzymes catalysent décroit. Et chez les animaux scorbutiques, les teneurs cardiaques et squelettiques en carnitine sont diminuées, alors que dans le foie et le rein elles restent inchangées et que dans le plasma elles sont augmentées (Hughes, Hurley et Jones, 1980 ; Nelson et al., 1981). Ces résultats s'expliquent par une baisse de la synthèse de carnitine en absence de vitamine C. En effet, le rein d'un cobaye témoin convertit 8 à 10 fois plus de triméthyllysine en triméthylaminobutyrate que ne le fait celui d'un cobaye scorbutique.

Chez le rat, on a tenté d'évaluer la quantité de carnitine produite. Dans le foie perfusé, elle serait de $0,12 \mu \mathrm{mol} / \mathrm{g}$ de foie frais $/ \mathrm{h}$, chez l'animal à jeûn (Zaspel, Sheridan et Henderson, 1980). In vivo, la production journalière de carnitine serait de $3 \mu \mathrm{mol}$. $(2 \mu \mathrm{mol} / 100 \mathrm{~g}$ de rat) selon Cederblad et Lindstedt (1976), de $30 \mu \mathrm{mol}(0.15 \mu \mathrm{mol} / \mathrm{g}$ de foie/h) selon Christiansen et Bremer (1976). Pour ces derniers auteurs, la production hépatique est suffisante pour assurer totalement le turnover de la carnitine chez le rat. Et de nombreux nutritionnistes pensent qu'il en est de même pour l'homme, en argumentant qu'il n'a jamais été possible de montrer que la carnitine est une substance nutritive indispensable.

\section{Synthèse lors du jeûne.}

Les teneurs de carnitine totale ont été suivies chez le rat soumis à un jeûne prolongé (Brass et Hoppel, 1978). Dans le plasma, elles diminuent au cours des 24 premières heures, puis elles augmentent régulièrement. Dans le foie, elles doublent après $18 \mathrm{~h}$ de privation de nourriture. Dans les muscles, elles augmentent faiblement, mais régulièrement. Compte-tenu de la masse musculaire, cette dernière observation pouvait impliquer une forte accélération de la synthèse de 
carnitine pendant le jeûne (Snoswell et Mclntosh, 1974). Mais Brass et Hoppel (1978), en montrant que le pool total de carnitine de l'organisme ne varie pas tout au long de la période de jeûne et que par ailleurs, l'élimination urinaire diminue, réfutent cette hypothèse.

\section{Synthèse lors de la période néonatale.}

Les résultats de Penn, Schmidt-Sommerfeld et Wolf (1980a, b) et de Schiff et al. (1979) montrent que les nouveaux-nés et les prématurés en particulier, ne sont pas capables de synthétiser assez de carnitine pour maintenir leur taux sanguin à un niveau suffisant.

Dans le foie de rat, on a pu montrer que l'activité de la $\gamma$-butyrobétaine hydroxylase, très faible chez le fœutus, augmente après la naissance et atteint sa valeur adulte à l'âge de 8 jours (Hahn, 1981). Ce n'est qu'après une semaine de vie que le jeune rat peut couvrir intégralement ses besoins en carnitine, sans recourir à un apport exogène. Chez l'homme, la situation semble comparable. A l'âge de 3 mois, l'activité de la $\gamma$-butyrobétaine hydroxylase hépatique ne représente que 12 p. 100 de celle de l'adulte; à l'âge de 2 à 5 ans, 30 p. 100 et à 15 ans, 100 p. 100 (Rebouche, 1980 ; Rebouche et Engel, 1980c).

Notons par ailleurs que chez le rat, l'activité de la protéine méthylase III augmente avec l'âge (Paik, Lee et Lawson, 1971).

\section{Synthèse lors d'une déficience en carnitine.}

Les déficiences primaires en carnitine peuvent être classées en deux groupes suivant qu'elles sont myopathiques ou systémiques. Lors d'une déficience myopathique, on observe une chute des taux musculaires en carnitine, qui serait due à un trouble du transport de la molécule du sang vers le muscle.

La déficience systémique est une maladie décrite pour la première fois par Karpati et al. (1975). Elle se caractérise par un effondrement de la carnitine musculaire et hépatique, une myopathie avec accumulation lipidique et fréquemment, une faiblesse musculaire et une myoglobinurie. Un ralentissement de la synthèse de carnitine avait été avancé pour expliquer cette déficience (Karpati et al., 1975 ; Boudin, 1976 ; Engel, Banker et Eiben, 1977).

Mais chez deux de ces malades, Rebouche et Engel (1981) viennent de démontrer que la $\mathrm{Me}_{3}$-lysine est parfaitement incorporée dans la carnitine. Précédemment, les mêmes auteurs (Rebouche et Engel, 1980b) avaient signalé que l'activité des 4 enzymes intervenant. dans la transformation de la $\mathrm{Me}_{3}$-lysine en carnitine était sensiblement la même chez des sujets présentant une déficience en carnitine que chez des sujets normaux. Pensant qu'un déficit du côté de la protéine méthylase III ou du côté de la $\mathrm{S}$-adenosylméthionine aurait des conséquences graves que l'on observe pas chez ces malades, Rebouche et Engel (1981) admettent que ces deux cas de déficience en carnitine ne s'expliquent pas par un déficit de la synthèse de carnitine.

\section{Conclusion.}

Au terme de cette étude bibliographique, il faut convenir que bien des lacunes existent encore dans notre connaissance sur la synthèse de la carnitine. Cer- 
tes, les différentes étapes qui conduisent de la lysine et de la méthionine à la carnitine, en passant par les principaux intermédiaires que sont la triméthyllysine et la $\gamma$-butyrobétaine, sont bien élucidées. Tout au plus subsiste-t'il quelques doutes sur l'origine de la triméthyllysine.

Certes, la localisation tissulaire des enzymes impliquées est maintenant bien définie et l'on sait qu'il existe dans ce domaine une grande variabilité d'une espèce animale à l'autre. Néanmoins, il n'est pas toujours possible de fixer avec exactitude le rôle et l'importance respectifs de chaque tissu dans la synthèse de la carnitine. Le schéma conçu à partir d'expériences réalisées chez le rat, qui implique que le rein assure la transformation de la triméthyllysine en $\gamma$ butyrobétaine et que le foie est seul capable d'hydroxyler cette dernière en carnitine n'est plus accepté, ni chez cet animal, ni surtout chez l'homme. La réalité, plus complexe, reste insaisissable. On ne sait pas non plus dans quelle mesure la carnitine apportée par l'alimentation, influence le taux de carnitine synthétisée par l'organisme.

Quant à la régulation de la biosynthèse de la carnitine, bien des travaux sont encore nécessaires. Les plus récents travaux de Rebouche et Engel (1981) suggèrent cependant que la déficience systémique observée dans certaines myopathies ne s'explique pas par un déficit de synthèse de la carnitine.

Recu en octobre 1981.

Accepté en février 1982.

\section{Références}

ANONYME, 1978. Deficiency of carnitine and its pathway of biosynthesis. Nutr. Rev., 36 , 305-309.

ANONYME, 1981. Carnitine biosynthesis in rat and man. Tissue specificity. Nutr. Rev., 39, $24-26$. BØHMER T., 1974. Conversion of butyrobetaine to carnitine in the rat in vivo. Biochim. biophys. Acta, 343, 551-557.

BORUM P., BROQUIST H. P., 1977a. Purification of S-Adenosylmethionine : $\varepsilon$-N-L-lysine methyltransferase. J. biol. Chem., 252, 5651-5655.

BORUM P. R., BROQUIST H. P., 1977b. Lysine deficiency and carnitine in male and female rats. J. Nutr., 107, 1209-1215.

BOUDIN G., 1976. Fatal systemic carnitine deficiency with lipid storage in skeletal muscle, heart, liver and kidney. J. neurol. Sci., 30, 313-325.

BRASS E. P., HOPPEL C. L., 1978. Carnitine metabolism in the fasting rat. J. biol. Chem., 253, 2688-2693.

BREMER J., 1961. Biosynthesis of carnitine in vivo. Biochim. biophys. Acta, 48, 622-624.

BREMER J., 1962. Carnitine precursors in the rat. Biochim. biophys. Acta, 57, 327-335.

BROQUIST H. P., 1980. Carnitin biosynthesis in Neurospora crassa, 7-17. In FRENKEL R. A., McGARRY J. D., Carnitine biosynthesis, metabolism and functions. Acad. Press, New York. BROQUIST H. P., BORUM P. R., 1977. Some aspects of carnitine nutriture. Compr. Ther., 3, 66-72. BROQUIST H. P., TANPHAICHITR V., 1973. Site of carnitine (CAR) biosynthesis in the rat. Fed. Proc., 32, 884.

CARTER A. L., FRENKEL R., 1979. The role of the kidney in the biosynthesis of carnitine in the rat. J. biol. Chem., 254, 10670-10674. 
CASILLAS E. R., ERICKSON B. J., 1975. Studies on carnitine synthesis in the rat epididymis. $J$. Reprod. Fert., 44, 287-291.

CEDERBLAD G., HOLM J., LINDSTEDT G., LINDSTEDT S., NORDIN I., SCHERSTEN T., $1979 . \quad \gamma-$ butyrobetaine hydroxylase activity in human and ovine liver and skeletal muscle tissue. Febs Lett., 98, 57-60.

CEDERBLAD G., LINDSTEDT S., 1976. Metabolism of labeled carnitine in the rat. Arch. Biochem. Biophys., 175, 173-180.

CHRISTIANSEN R. Z., BREMER J., 1976. Active transport of butyrobetaine and carnitine into isolated liver cells. Biochim. biophys. Acta, 448, 562-577.

COX R. A., HOPPEL C. L., 1973a. Biosynthesis of carnitine and 4-N-trimethylaminobutyrate from lysine. Biochem. J., 136, 1075-1982.

COX R. A., HOPPEL C. L., 1973b. Biosynthesis of carnitine and 4-N-trimethylaminobutyrate from 6-N-trimethyllysine. Biochem. J., 136, 1083-1090.

COX R. A., HOPPEL C. L., 1974a. Carnitine biosynthesis in rat liver slices. Biochem. biophys. Acta, 362, 403-413.

COX R. A., HOPPEL C. L., 1974b. Carnitine and trimethylaminobutyrate synthesis in rat tissues. Biochem. J., 142, 699-701.

ENGEL A. G., ANGELINI C., 1973. Carnitine deficiency of human skeletal muscle with associated lipid storage myopathy: a new syndrome. Science, 179, 899-902.

ENGEL A. G., BANKER B. Q., EIBEN R. M., 1977. Carnitine deficiency : clinical, morphological and biochemical observations in a fatal case. J. Neurol. Neurosurg. Psych., 40, 313-322.

ENGLARD S., 1979. Hydroxylation of $\gamma$-butyrobetaine to carnitine in human and monkey tissues. Febs Lett., 102, 297.

ENGLARD S., CARNICERO H. H., 1978. $\gamma$-butyrobetaine hydroxylation to carnitine in mammalian kidney. Arch. Biochem. Biophys., 190, 361-364.

ENGLARD S., HORWITZ L. J., MILLS J. T., 1978. A simplified method for the measurement of $\gamma$-butyrobetaine hydroxylase activity. J. Lip. Res., 19, 1057-1063.

ERFLE J. D., 1975. Hydroxylation of $\gamma$-butyrobetaine by rat and ovine tissues. Biochim. biophys. Res. Comm., 64, 553-557.

FRAENKEL G., 1953. Studies on the distribution of vitamin $B_{T}$ (carnitine). Biol. Bull., 104, 359-371.

FRAENKEL G., FRIEDMAN S., 1957. Carnitine. In HARRIS P., MARRIAN G., THIMANN K., Vitamins and hormones, vol. 15, 73-118. Acad. Press, New York.

FRENKEL R. A., CARTER A. L., 1980. Synthesis of carnitine precursors in rat kidney, 19-33. In FRENKEL R. A., McGARRY J. D., Carnitine biosynthesis, metabolism and functions. Acad. Press, New York.

FRITZ I. B., 1955. Effects of muscle extracts on the oxidation of palmitic acid by liver slices and homogenates. Acta physiol. scand., 34, 367-385.

HAHN P., 1981. The development of carnitine synthesis from $\gamma$-butyrobetaine in the rat. Life Sci, 28, 1057-1060.

HAIGLER H. T., BROQUIST H. P., 1974. Carnitine synthesis in rat tissue slices. Biochem. biophys. Res. Comm., 56, 676-681.

HENDERSON L. M., HULSE J. H., HENDERSON L. L., 1980. Purification of the enzymes involved in the conversion of trimethyllysine to trimethylaminobutyrate, 35-43. In FRENKEL R. A., McGARRY J. D., Carnitine biosynthesis, metabolism and functions. Acad. Press, New York.

HOCHALTER J. D., HENDERSON L. M., 1976. Carnitine biosynthesis : the formation of glycine from carbons 1 and 2 of 6-N-trimethyl-L-lysine. Biochem. biophys. Res. Comm., 70, 364-366.

HOPPEL C. L., COX R. A., NOVAK R. F., 1980. $\mathrm{N}^{6}$-trimethyl-lysine metabolism. 3-hydroxy$\mathrm{N}^{6}$-trimethyl-lysine and carnitine biosynthesis. Biochem. J., 188, 509-519.

HOPPEL C. L., NOVAK R., COX R. A., 1976. 6-N-trimethyllysine metabolism and carnitine biosynthesis. Fed. Proc., 35, 1478.

HORNE D. W., BROQUIST H P., 1973. Role of lysine and $\varepsilon-\mathrm{N}$-trimethyllysine in carnitine biosynthesis. 1. Studies in Neurospora crassa. J. biol. Chem., 248, 2170-2175.

HORNE D. W., TANPHAICHITR V., BROQUIST H. P., 1971. Role of lysine in carnitine biosynthesis in Neurospora crassa. J. biol. Chem., 246, 4373-4375.

HUGHES R. E., HURLEY R. J., JONES E., 1980 . Dietary ascorbic acid and muscle carnitine $[\beta-\mathrm{OH}-\gamma$-(trimethylamino)butyric acid] in guinea-pigs. Br. J. Nutr., 43, 385-387. 
HULSE J. D., ELLIS S. R., HENDERSON L. M., 1978. Carnitine biosynthesis. $\beta$-hydroxylation of trimethyllysine by an $\alpha$-ketoglutarate-dependent mitochondrial dioxygenase. $J$. biol. Chem., 253, 1654-1659.

HULSE J. D., HENDERSON L. V. M., 1980. Carnitine biosynthesis. Purification of 4-N-trimethylaminobutyraidehyde dehydrogenase from beef liver. J. biol. Chem., 255, 1146-1151.

KARPATI G., CARPENTER S., ENGEL A. G., WATTERS G., ALLEN J., ROTHMAN S., KLASSEN G., MAMER O. A., 1975. The syndrome of systemic carnitine deficiency. Clinical, morphologic, biochemical and patho-physiologic features. Neurology, 25, 16-24.

KAUFMAN R. A., BROQUIST H. P., 1977. Biosynthesis of carnitine in Neurospora crassa. J. biol. Chem., 252, 7437-7439.

KHAIRALLAH E. A., WOLF G., 1965. Growth-promoting and lipotropic effect of carnitine in rats fed diets limited in protein and methionine. J. Nutr., 87, 469-476.

KHAN L., BAMJI M. S., 1977. Plasma carnitine levels in children with protein-calorie malnutrition before and after rehabilitation. Clin. chim. Acta, 75, 163-166.

KHAN L., BAMJI M. S., 1979. Tissue carnitine deficiency due to dietary lysine deficiency : triglyceride accumulation and concomitant impairment in fatty acid oxidation. $J$. Nutr., 109, 24-31.

KRZYSIK B., VERGNES J. P., McMANUS I. R., 1971. Enzymic methylation of skeletal muscle contractile protein. Arch. Biochem. Biophys., 146, 34-45.

LABADIE J., DUNN W. A., ARONSON N. N., 1976. Hepatic synthesis of carnitine from proteinbound trimethyllysine. Lysosomal digestion of methyl-lysine labelled asialofetuin. Biochem. J., 160, 85-95.

LINDSTEDT G., 1967. Hydroxylation of $\gamma$-butyrobetaine to carnitine in rat liver. Biochemistry, 6. $1271-1282$.

LINDSTEDT G., LINDSTEDT S., 1961. On the biosynthesis and degradation of carnitine. Biochem. biophys. Res. Comm., 6, 319-323.

LINDSTEDT G., LINDSTEDT S., 1962. On the hydroxylation of $\gamma$-butyrobetaine to carnitine in vitro. Biochem. biophys. Res. Comm., 7, 394-397.

LINDSTEDT G., LINDSTEDT S., 1970. Cofactor requirements of $\gamma$-butyrobetaine hydroxylase from rat liver. J. biol. Chem., 245, 4178-4186.

LINDSTEDT G., LINDSTEDT S., NORDIN I., 1980. Hydroxylation of $\gamma$-butyrobetaine, 45-56. In FRENKEL R. A., McGARRY J. D., Carnitine biosynthesis, metabolism and functions. Acad. Press, New York.

LINNEWEH W., 1929. Zur Kenntnis des Carnitins. Hoppe-Seyler's Z. physiol. Chem., 182, 8-12.

MIKHAIL M. M., MANSOUR M. M., 1976. The relationship between serum carnitine levels and the nutritional status of patients with schistosomiasis. Clin. chim. Acta, 71, 207-214.

MITCHELL M. E., 1978. Carnitine metabolism in human subjects. I. Normal metabolism. Am. J. clin. Nutr., 31, 293-306.

NELSON P. J., PRUITT R. E., HENDERSON L. L., JENNESS R., HENDERSON L. M., 1981. Effect of ascorbic acid deficiency on the in vivo synthesis of carnitine. Biochim. biophys. Acta, 672, 123-127.

PAIK W. K., LEE H. W., LAWSON D., 1971. Age-dependency of various protein methylases. Exp. Gerontol., 6, 271-277.

PAIK W. K., KIM S., 1971. Protein methylation. Science, 174, 114-119.

PAIK W. K., KIM S., 1975. Protein methylation : chemical, enzymological and biological significance. Adv. Enzymol., 42, 227-286.

PAIK W. K., NOCHUMSON S., KIM S., 1977. Carnitine biosynthesis via protein methylation. Trends biochem. Sci., 2, 159-161.

PENN D., SCHMIDT-SOMMERFELD E., WOLF H., 1980a. Carnitine deficiency in premature infants receiving total parenteral nutrition. Europ. J. Pediat., 133, 181.

PENN D., SCHMIDT-SOMMERFELD E., WOLF H., 1980b. Carnitine deficiency in premature infants receiving total parenteral nutrition. Early human development, 4, 23-34.

REBOUCHE C. J., 1980. Comparative aspects of carnitine biosynthesis in microorganisms and mammals with attention to carnitine biosynthesis in man, 57-69. In FRENKEL R. A., McGARRY J. D., Carnitine biosynthesis, metabolism and functions. Acad. Press, New York. 
REBOUCHE C. J., BROQUIST H. P., 1976. Carnitine biosynthesis in Neurospora crassa : enzymatic conversion of lysine to $\varepsilon-\mathrm{N}$-trimethyllysine. J. Bacteriol., 126, 1207-1214.

REBOUCHE C. J., ENGEL A. G., 1980a. Significance of renal $\gamma$-butyrobetaine hydroxylase for carnitine biosynthesis in man. J. biol. Chem., 255, 8700-8705.

REBOUCHE C. J., ENGEL A. G., 1980b. In vitro analysis of hepatic carnitine biosynthesis in human systemic carnitine deficiency. Clin. chim. Acta, 106, 295-300.

REBOUCHE C. J., ENGEL A. G., 1980c. Tissue distribution of carnitine biosynthetic enzymes in man. Biochim. biophys. Acta, 630, 22-29.

REBOUCHE C. J., ENGEL A. G., 1981. Primary systemic carnitine deficiency : I. Carnitine biosynthesis. Neurology, 31, 813-818.

SACHAN S., HOPPEL C. L., 1980. Carnitine biosynthesis. Hydroxylation of $\mathrm{N}^{6}$-trimethyl-lysine to 3-hydroxy- $\mathrm{N}^{6}$-trimethyl-lysine. Biochem. J., 188, 529-534.

SCHIFF D., CHAN G., SECCOMBE D., HAHN P., 1979. Plasma carnitine levels during intravenous feeding of the neonate. J. Pediat, 95, 1043-1046.

SNOSWELL A. M., McINTOSH G. H., 1974. The liver as the site of carnitine biosynthesis in sheep with alloxan-induced diabetes. Aust. J. biol. Sci, 27, 645-650.

SOLBERG H. E., 1971. Carnitine octanoyl transferase. Evidence for a new enzyme in mitochondria. Febs Lett., 12, 134-136.

STRENGTH D. R., YU S. Y., DAVIS E. Y., 1965. Biosynthesis of carnitine; dietary factors that influence concentration of carnitine in tissues, 45-56. In WOLF G., Recent research on carnitine. MIT Press, Cambridge.

TANPHAICHITR V., BROQUIST H. P., 1973a. Lysine deficiency in the rat : concomitant impairment in carnitine biosynthesis. J. Nutr., 103, 80-87.

TANPHAICHITR V., BROQUIST H. P., 1973b. Role of lysine and $\varepsilon$-N-trimethyllysine in carnitine biosynthesis. J. biol. Chem., 248, 2176-2181.

TANPHAICHITR V., BROQUIST H. P., 1974. Site of carnitine biosynthesis in the rat. J. Nutr., 104, 1669-1673.

TANPHAICHITR V., HORNE D. W., BROOUIST H. P., 1971. Lysine, a precursor of carnitine in the rat. J. Biol. Chem., 246, 6364-6366.

TANPHAICHITR V., ZAKLAMA M. S., BROQUIST H. P., 1973. Dietary lysine (lys) and carnitine (CAR) : relation to growth and fatty liver in rats. Fed. Proc., 32, 884.

TANPHAICHITR V., ZAKLAMA M. S., BROQUIST H. P., 1976. Dietary lysine and carnitine : relation to growth and fatty livers in rats. $J$. Nutr., 106, 111-117.

WOLF G., BERGER C. R. A., 1961. Studies on the biosynthesis and turnover of carnitine. Arch. Biochem. Biophys., 92, 360-365.

ZASPEL B. J., HENDERSON L. M., 1979. Metabolism and transport precursors in various organs of the rat. Fed. Proc., 38, 280.

ZASPEL B. J., SHERIDAN L. J., HENDERSON L. M., 1980. Transport and metabolism of carnitine precursors in various organs of the rat. Biochim. biophys. Acta, 631, 192-202. 